\title{
Literacy-Sensitive Intervention for COPD Patients
}

\author{
Katie Kiser, PharmD, $\mathrm{BCPS}^{7}$, Daniel Jonas, $\mathrm{MD}, \mathrm{MPH}^{2}$, and Darren Dewalt, $\mathrm{MD}, \mathrm{MPH}{ }^{2}$ \\ 'University of Maryland School of Pharmacy, Baltimore, MD, USA; ${ }^{2}$ Division of General Internal Medicine, University of North Carolina School of \\ Medicine, Chapel Hill, NC, USA.
}

$\mathrm{J}$ Gen Intern Med 27(9): 1104

DOI: $10.1007 / \mathrm{s} 11606-012-2084-7$

(c) Society of General Internal Medicine 2012

The Authors Reply:-We thank Shapiro et al. for their comments concerning what role poor vision might have played in the results of the Short Test of Functional Health Literacy in Adults (S-TOFHLA) and thus classification of participants' health literacy in our study-"A Randomized Controlled Trial of a Literacy-Sensitive Self-Management Intervention for Chronic Obstructive Pulmonary Disease Patients". ${ }^{1}$ We would like to clarify that we used the large print (14-point font) version of the S-TOFHLA and did not assess the participant's vision or if they used corrective lenses before administering the S-TOFHLA. We did allow patients to opt out if they indicated their vision was not sufficient to take the test. During development and validation of the S-TOFHLA, participants with 20/70 to $20 / 100$, which is classified as moderate vision loss based on ICD-9-CM, were administered the large print version and those with worse vision were excluded (three percent were excluded for vision worse than 20/100). ${ }^{2}$ In lay terms, a visual acuity of 20/100 means a person would require letters to be five times larger or closer to be able to read compared to $20 / 20$ vision. Press et al. assessed participant's vision in their study and classified vision as insufficient to complete the S-TOFHLA (large print version) if their acuity scores for both eyes were worse than $20 / 50 .^{3}$ Although the cut point for poor vision is more stringent than required by the developers of the S-TOFHLA, such a high rate of poor vision (29\% in Press et al.) is an important clinical finding with important implications. Other estimates of poor vision are much lower $(1.98 \%$ of Americans older than age 40 have best corrected vision worse than 20/ 40). ${ }^{4}$ It is possible that the inpatient location of the Press et al. study contributed to the high prevalence of poor vision. Since we did not screen vision, we are unable to definitively say that vision did not affect our S-TOFHLA results.

We agree with Press and colleagues that poor vision may be an underappreciated factor for many patients managing chronic diseases and that poor vision alone or in conjunction with low health literacy may require alternative interventions to improve outcomes. Another concern is that patients may have the visual acuity necessary to complete the S-TOFHLA (large print version), but have barriers to other elements of their health care such as reading prescription bottles or patient education handouts that may not have sufficient font style or size for their vision.

Corresponding Author: Katie Kiser, PharmD, BCPS; University of Maryland School of Pharmacy, 20 N. Pine Street, Baltimore, MD 21201, USA (e-mail: kkiser@rx.umaryland.edu).

\section{REFERENCES}

1. Kiser K, Jonas D, Warner Z, Scanlon K, Bryant Shilliday B, DeWalt D. A randomized controlled trial of a literacy-sensitive self-management intervention for chronic obstructive pulmonary disease patients. J Gen Intern Med. 2012;27(2): 190-5.

2. Baker DW, Williams MV, Parker RM, Gazmararian JA, Nurss J. Development of a brief test to measure functional health literacy. Patient Educ Couns. 1999;38:33-42.

3. Press VG, Arora VM, Shah LM, et al. Misuse of respiratory inhalers in hospitalized patients with asthma or COPD. J Gen Intern Med. 2011;26 (6):635-42.

4. Eye Diseases Prevalence Research Group. Causes and Prevalence of Visual Impairment Among Adults in the United States. Arch Ophthalmol. 2004;122:477-85. 